Research Article

\title{
Realistic Models of Financial Market and Structural Stability
}

\author{
Sergey N. Smirnov \\ Faculty of Computational Mathematics and Cybernetics, Lomonosov Moscow State University, Moscow 119991, Russia
}

Correspondence should be addressed to Sergey N. Smirnov; s.n.smirnov@gmail.com

Received 16 December 2020; Accepted 8 June 2021; Published 21 June 2021

Academic Editor: Basil Papadopoulos

Copyright (C) 2021 Sergey N. Smirnov. This is an open access article distributed under the Creative Commons Attribution License, which permits unrestricted use, distribution, and reproduction in any medium, provided the original work is properly cited.

The main aim of this article is to show the role of structural stability in financial modelling; that is, a specific "no-arbitrage" property is unaffected by small perturbations of the model's dynamics. We prove that under the structural stability assumption, given a convex compact-valued multifunction, there exists a stochastic transition kernel with supports coinciding with this multifunction and one that is strong Feller in the strict sense. We also demonstrate preservation of structural stability for sufficiently small deviations of transition kernels for different probability metrics.

\section{Introduction}

The main aim of this paper is to demonstrate the importance of structural stability for financial modelling. Structural stability is a fundamental property of a model and means that the qualitative behavior of the model is unaffected by small (in a certain sense) perturbations of its dynamics. (The term "structural stability" is borrowed from dynamical systems theory. Structurally stable systems were introduced in 1937 by Aleksandr Andronov and Lev Pontryagin [1] under the name "systèmes grossiers," or coarse systems. Unlike Lyapunov stability, related to perturbations of the initial conditions for a fixed system, structural stability deals with perturbations of the system itself.) In the context of economics, such qualitative behavior of a model of a financial market implies that it admits no "arbitrage," in some sense to be made precise. The idea of stability of the noarbitrage property under some perturbation of the model is, of course, present in the literature. We restrict ourselves to mentioning only a few related papers. Let us start with [2], where a "robust no-arbitrage condition" was introduced in the case of market friction (proportional transaction costs). The economic meaning of this notion is that there is still room for the broker to offer some discount in quoting bid and ask prices without creating an arbitrage possibility. There are also ad hoc definitions of the robust no-arbitrage property (in the framework of a particular model), for example, that by the authors of [3], who considered a case with nontradable options quoted with bid-ask spreads. In this set-up, the robust no-arbitrage property turns out to be equivalent to no arbitrage under the additional assumption that hedging options with nonzero spread are nonredundant. For a frictionless market, a simple, but nice, result is presented in [4], where it is shown that for a nonredundant one-step model, the no-arbitrage property is preserved under sufficiently small perturbations of the initial probability in the total variation metric. Sometimes, such properties are used implicitly, as in [5], who considered a continuous-time model of a financial market with primary assets, options that are nontradable except at the initial time moment, and continuously traded European options. The general duality results of that paper exploited its Assumption 3.1 concerning these options, requiring that calibrated martingale measures exist under arbitrarily small perturbations of the initial prices. Thus, depending on the context, structural stability can be formalised in various ways. In any case, it is of major importance for the correct numerical solution of a financial engineering problem.

Here, we propose a formalisation of structural stability in the framework of a guaranteed deterministic approach. A sufficiently detailed exposition of this approach can be found in [6], but for convenience, we give a brief introduction in Section 2, with a summary of the notions involved and the results necessary for the developments in the rest of the 
present paper. The main premise of this approach is based on a specific assumption concerning a priori information regarding price movements. The model of the market represents "uncertain" price dynamics with discrete time and can be considered as purely deterministic: the initial problem setting does not use any probability or family of probabilities. We should stress here that we adopt an alternative interpretation to the common robust approach to pricing of contingent liabilities. Our interpretation of superhedging is game-theoretic: we deal with a deterministic dynamic two-player zero-sum game of "hedger" against "market". (With regard to the superhedging problem, the cornerstone of the guaranteed deterministic approach is a recurrence relation given by the Bellman-Isaacs equations, which can be derived directly in an economic context by choosing at step $t$ the "best" admissible hedging strategy $h \in D_{t}(\cdot) \subseteq \mathbb{R}^{n}$ for the "worst" scenario of (discounted) price increments $y \in K_{t}(\cdot)$ for the given functions $g_{t}(\cdot)$ describing the potential payouts on the option. We share an idea, suggested in unpublished work of Carassus and Varioglu about 15 years ago and finally published in [7]: in order to get a meaningful theory, it is reasonable to assume the boundedness of price increments, so usually $K_{t}(\cdot)$ is assumed to be compact. A family of probabilities appears as a secondary notion, thanks to the introduction of mixed strategies of the "market." However, this material (concerning the Bellman-Isaacs equations and the corresponding mixed strategies) is not needed for the present paper; interested readers can refer, for example, to [6].) Note that we deliberately do not use the mathematical machinery of probability theory and stochastic processes $(\sigma$-algebras, measurability, filtrations, etc.) at least in the formulation of our results. In this way, we simplify the presentation, avoiding unnecessary assumptions and technicalities, and achieve greater transparency from an economic point of view (at least concerning the formulation of the results). In our opinion, all the delicate mathematical problems dealing with measurability issues are of a completely set-theoretic nature and therefore have nothing to do with modelling from a practical point of view.

One of the first publications to develop a guaranteed deterministic approach is that by Vasiliy Kolokoltsov [8]. To the best of our knowledge, this was the first work to explicitly articulate this approach to pricing and hedging contingent clams. The guaranteed deterministic approach is closely related to a class of market models called interval models in [9], especially to the ideas and results of Kolokoltsov published in Chapters 11-14 of the latter reference, including the independent discovery of the game-theoretic interpretation of risk-neutral probabilities under the assumption of no trading constraints; we find this interpretation to be quite important from an economic point of view.

\section{Preliminaries}

2.1. Financial Market Model in the Deterministic Framework. Let us first describe the financial market model in the deterministic framework. Consider discounted prices of $n$ risky assets; without loss of generality, we can suppose that a riskfree asset has a fixed price equal to 1 and so in what follows, we call "discounted prices" simply "prices." Let $X_{t}$ be the price vector at time $t$ and $\Delta X_{t}=X_{t}-X_{t-1}$ be the vector of price increments. The abovementioned assumption about a priori information describing the uncertainty of price movements is as follows: the price increments $\Delta X_{t}$ lie in a priori given closed (as we have already mentioned above, a more appropriate assumption is the compactness of $K_{t}(\cdot)$; see [7]) sets (The dot “." indicates the variables representing the price evolution. More precisely, it indicates the prehistory $\bar{x}_{t-1}=\left(x_{0}, \ldots, x_{t-1}\right) \in\left(\mathbb{R}^{n}\right)^{t}$ for $K_{t}$, and it indicates the history $\bar{x}_{t}=\left(x_{0}, \ldots, x_{t}\right) \in\left(\mathbb{R}^{n}\right)^{t+1}$ for the functions $v_{t}^{*}$ and $g_{t}$ introduced below.) $K_{t}(\cdot) \subseteq \mathbb{R}^{n}, t=1, \ldots, N$. We denote by $B_{t}$ the set of possible trajectories (or paths) of asset prices in the time interval $[0, t]$, i.e.,

$$
B_{t}=\left\{\left(x_{0}, \ldots, x_{t}\right): x_{0} \in K_{0}, \Delta x_{1} \in K_{1}\left(x_{0}\right), \ldots, \Delta x_{t} \in K_{t}\left(x_{0}, \ldots, x_{t-1}\right)\right\}
$$

In general, we suppose that there are trading constraints, concerning only risky assets. These are described by a priori given sets $D_{t}(\cdot) \subseteq \mathbb{R}^{n}, t=1, \ldots, N$, assumed to be convex and such that $0 \in D_{t}(\cdot)$. An admissible hedging strategy at time step $t$ is therefore $h \in D_{t}(\cdot)$.

2.2. Notions of "No Arbitrage". The different notions of "no arbitrage" in the framework of the deterministic market model can be relevant (Two notions of arbitrage are introduced below: deterministic arbitrage opportunity (DAO) coincides with "one-point arbitrage" and in our setting is also equivalent to the quasi-sure arbitrage of [10], while deterministic sure arbitrage (DSA) coincides with "strong arbitrage," to use the unified terminology of robust modelling in [11]. A detailed analysis of the relation between different "no-arbitrage" notions in the framework of robust modelling of financial markets in discrete time is presented in [12].) and geometric criteria (in terms of convex hulls of $\left.K_{t}(\cdot)\right)$ are obtained. For the convenience of the reader, we give several definitions of the notions.

Definition 1. By a deterministic arbitrage opportunity (DAO) at time step $t$, we mean that there exists a strategy $h^{*} \in D_{t}(\cdot)$ such that $h^{*} y \geq 0$ for all $y \in K_{t}(\cdot)$ and there exists a price movement $y^{*} \in K_{t}(\cdot)$ such that $h^{*} y^{*}>0$.

Definition 2. By deterministic sure arbitrage (DSA) at time step $t$, we mean that there exists a strategy $h^{*} \in D_{t}(\cdot)$ such that $h^{*} y>0$ for all $y \in K_{t}(\cdot)$.

Definition 3. We say that there is deterministic sure arbitrage with unlimited profit (DSAUP) at time step $t$ if the 
function (This can be interpreted as the guaranteed profit for a strategy $h \in D_{t}(\cdot)$. Note that if the sets $K_{t}(\cdot)$ are compact, then the infimum becomes minimum, that is, $\min \left\{h y, y \in K_{t}(\cdot)\right\}$, and is attained for some (least profitable scenario) $y_{h}^{*} \in K_{t}(\cdot)$; thus, it is positive for any deterministic sure arbitrage strategy $h^{*} \in D_{t}(\cdot)$.) $h \longmapsto \inf \left\{h y, y \in K_{t}(\cdot)\right\}$ takes arbitrarily large values for $h \in D_{t}(\cdot)$.

Using these three notions of "arbitrage," we can define the corresponding "no-arbitrage" properties on a time interval: each "no-arbitrage" property on a time interval is tantamount to a "no-arbitrage" property at every time step $t \in\{1, \ldots, N\}$ of this interval for any price prehistory $\bar{x}_{t-1}=\left(x_{0}, \ldots, x_{t-1}\right) \in B_{t-1}$. Hence, we consider the following "no-arbitrage" properties: no deterministic arbitrage opportunity (NDAO), no deterministic sure arbitrage (NDSA), and no deterministic sure arbitrage with unbounded profit (NDSAUP).

2.3. Structural Stability. According to our interpretation, $K_{t}(\cdot)$ reflects the agent's beliefs about price movements, which are naturally inexact. (On the other hand, the trading constraints are supposed to be defined exactly.) In particular, the proposed approach can arise in the framework of fuzzy (or hybrid probabilistic-fuzzy) models; for example, the uncertainty of price movement can be formalised by a system of fuzzy sets (A fuzzy set $A$, subset of $X$ (called universe), can be defined by means of its membership function $x \longmapsto \mu_{A}(x) \in[0,1], x \in X$. Given $\alpha \in[0,1]$, the super-level set of $\mu_{A}$ corresponding to $\alpha$, i.e., the (crisp) set $\left\{x \in X: \mu_{A}(x) \geq \alpha\right\}$ is called the $\alpha$-cut of the fuzzy set $\mathrm{A}$ (for more details, we refer the reader to [13]).) $A_{t}(x), x \in B_{t-1}$ with upper semicontinuous membership functions, defined on the universe $\mathbb{R}^{n}$, and $K_{t}(\cdot)$ can be chosen as $\alpha$-cut of the fuzzy set $A_{t}(\cdot)$ for some $\alpha \in[0,1]$.

Since our interpretation appeals to a vague knowledge about the price behaviour, this explains the importance of structural stability, which in our context means that a specific "no-arbitrage" property is unaffected by perturbations of $K_{t}(\cdot)$ that are sufficiently small with respect to the Pompeiu-Hausdorff metric $d_{\mathrm{PH}}$; we call such a "no-arbitrage" property robust or coarse. In what follows, we consider two coarse "no-arbitrage" properties: robust no deterministic arbitrage opportunity (RNDAO) and robust no deterministic sure arbitrage with unlimited profit (RNDSAUP). More precisely, it is defined below.

Definition 4. Suppose that the initial market model satisfies the NDAO (respectively, NDSAUP) condition and consider a perturbed model with increments $\Delta X_{t}$ lying in compact sets $\widetilde{K}_{t}(\cdot)$ for $t=1, \ldots, N$. The robust (or coarse) NDAO (respectively, NDSAUP) property, abbreviated RNDAO (respectively, RNDSAUP), means that for any $t \in\{1, \ldots, N\}$ and any prehistory of the prices $x \in B_{t-1}$, there exists $\varepsilon_{t}(x)>0$ such that if $d_{\mathrm{PH}}\left(\widetilde{K}_{t}(x), K_{t}(x)\right) \leq \varepsilon_{t}(x)$, then the perturbed market model still satisfies the NDAO (respectively, NDSAUP) condition.

The notions of "no arbitrage" have geometric criteria in terms of conv $\left(K_{t}(\cdot)\right)$. Here, we mention only two of them, which are of interest in the case of no trading constraints, (The case with trading constraints and the corresponding geometrical condition of RNDSAUP is studied in [14]. Note that, in general, the condition RNDSAUP does not imply $\operatorname{int}\left(\operatorname{conv}\left(K_{t}(\cdot)\right)\right) \neq \varnothing$.) i.e., when $D_{t}(\cdot) \equiv \mathbb{R}^{n}$. The condition $\mathrm{NDAO}$ is equivalent to the Jacod-Shiryaev geometric criterion: (In the probabilistic setting, this geometric criterion (understood almost surely) was found by Jacod and Shiryaev [15].) $0 \in \operatorname{ri}\left(\operatorname{conv}\left(K_{t}(\cdot)\right)\right), t=1, \ldots, N$, and RNDAO is equivalent to the "enhanced" Jacod-Shiryaev geometric criterion $0 \in \operatorname{int}\left(\operatorname{conv}\left(K_{t}(\cdot)\right)\right), t=1, \ldots, N$, where $\operatorname{conv}(A)$ is the convex hull of a set $A, \operatorname{ri}(A)$ is the relative interior of a convex set $A$, and $\operatorname{int}(A)$ is the interior of a set A.

2.4. Realistic Models. Let us switch for a while to considerations in the framework of the "probabilistic" approach. We regard as "realistic" a stochastic model of market behaviour if the conditional distributions of the current price depend "continuously" on the price history. This is a natural property of market models from an economic point of view: there are no economic grounds for discontinuity of the dependence on price prehistory. The following is a formalisation of this property.

Definition 5. We say that a stochastic model of price evolution is realistic, if the transition kernels $Q_{t}$ corresponding to the conditional probabilities of the price $X_{t} \in \mathbb{R}^{n}$ at time $t$ with a known history $\bar{X}_{t-1}=\bar{x}_{t-1} \in\left(\mathbb{R}^{n}\right)^{t}$ admit a version satisfying the Feller property, i.e., the mapping $x \mapsto P_{t}(x, \cdot)$ is continuous (For measurability issues, see Proposition 1 in [16].) in the weak topology on the space of probability measures.

Note that the deterministic and stochastic approaches lead to the same notions of "no arbitrage" in terms of $\operatorname{conv}\left(\operatorname{supp}\left(P_{t}(x, \cdot)\right)\right)$, when the reference probability measure is specified by means of the Ionescu-Tulcea theorem [17] using Feller transition kernels.

In the context of the deterministic approach, we have proposed the following formalisation of a "realistic" model of price evolution. Consider a financial market model with deterministic evolution of prices, where possible price increments at time $t$ are described by (In this case, $K_{t}(\cdot)$ are not necessarily compact (but closed).) $K_{t}(x)$, given price prehistory $x$; or, equivalently, possible price values at time $t$ are described by $F_{t}(x)=x+K_{t}(x)$.

Definition 6. We call this model "realistic" if there exist mixed market strategies $P_{t}(x, \cdot)$ representable as Feller transition kernels, (This can be interpreted as a smooth version of conditional distributions $X_{t}$, given prehistory $\bar{X}_{t-1}=x$.) satisfying the consistency condition, which is a relation between stochastic and deterministic models:

$$
\operatorname{supp}\left(P_{t}(x, \cdot)\right)=F_{t}(x), \quad x \in B_{t-1} \text { for } t=1, \ldots, N .
$$

A necessary and sufficient condition for the existence of such a selector $P_{t}$ is lower semicontinuity of the multifunctions $F_{t}$, as shown in Theorem 2 of [16]. In what follows, 
we prefer to deal with transition kernels (Obviously, the multifunctions $K_{t}(\cdot)$ and $F_{t}(\cdot)$ have the same continuity and measurability properties. The kernels $Q_{t}$ are Feller iff $P_{t}$ are Feller.) $Q_{t}(x, A)=P_{t}(x, x+A)$; in this case, the consistency condition (2) can be rewritten as

$$
\operatorname{supp}\left(Q_{t}(x, \cdot)\right)=K_{t}(x), \quad x \in B_{t-1} \text { for } t=1, \ldots, N .
$$

\section{Main Results}

3.1. Notations. Denote by $\sigma_{A}, \mathrm{cl}(A)$, and bd $(A)$, respectively, the support function, the closure, and the boundary of a set $A$. Denoting the Euclidean norm in $\mathbb{R}^{n}$ by $\|x\|_{2}$, let $\|A\|_{2}=\sup \left\{\|x\|_{2}: x \in A\right\}, \quad \rho(x, y)=\|x-y\|_{2}, \quad \rho(x, A)=$ $\inf \{\rho(x, y): y \in A\}, \quad B_{\delta}(y)=\left\{x \in \mathbb{R}^{n}:\|x-y\|_{2}<\delta\right\} \quad$ (the open ball of radius $\delta$ with centre at $y), \bar{B}_{\delta}(y)=\operatorname{cl}\left(B_{\delta}(y)\right)$, and $S_{\delta}(y)=\operatorname{bd}\left(B_{\delta}(y)\right)$ (the sphere of radius $\delta$ with centre at $y$ ). Denote by $\|\cdot\|_{T V}$ the total variation norm on the space of finite alternating measures, $I_{A}$ the indicator of a set $A, \mathscr{C}_{\mathbb{R}^{n}}$ the class of all nonvoid convex subsets of $\mathbb{R}^{n}, \mathscr{C}_{\mathbb{R}^{n}}^{*}$ the class of all nonvoid convex compact subsets of $\mathbb{R}^{n}$, and $\mathscr{C}_{\mathbb{R}^{n}}^{0}=\left\{K \in \mathscr{C}_{\mathbb{R}^{n}}^{*}: 0 \in K\right\}$.

For a monotone nondecreasing function $f:[0, \infty) \longrightarrow \mathbb{R}$, we define the upper generalised inverse by

$$
f^{\lfloor-1\rfloor}(y)=\inf \{x \in[0, \infty): f(x)>y\}=\sup \{x \in[0, \infty): f(x) \leq y\}
$$

which is nondecreasing right-continuous and the lower generalised inverse by

$$
f^{\lfloor-1\rfloor}(y)=\sup \{x \in[0, \infty): f(x)<y\}=\inf \{x \in[0, \infty): f(x) \geq y\}
$$

which is nondecreasing left-continuous. Evidently, $f^{\lfloor-1\rfloor}(y) \geq f^{\lfloor-1\rfloor}(y)$. If $f$ is strictly increasing, then $f^{\lfloor-1\rfloor}(y)=f^{\lfloor-1\rfloor}(y)$, and coinciding generalised inverse functions are continuous.

Denote $d(z, A)=\min _{l \in S_{1}(0)} \sigma_{A-z}(l)$ for a set $A \subseteq \mathbb{R}^{n}$ such that $\operatorname{conv}(A) \neq \mathbb{R}^{n}$. The quantity $d(z, A)$ can be characterised as an alternating distance from the point $z$ to the boundary of the convex hull of the set $A$ : negative for $z \notin \mathrm{cl}(\operatorname{conv}(A))$, zero for $z \in \mathrm{bd}(\operatorname{conv}(A))$, and, if $\operatorname{int}(\operatorname{conv}(A)) \neq \varnothing$, positive for $z \in \operatorname{int}(\operatorname{conv}(A))$.

For a compact $K \in \mathscr{C}_{\mathbb{R}^{n}}^{0}$, define a function $r$ by (Note that for such arguments, the function $r$ takes finite non-negative values.)

$$
r(K)=d(0, K)=\min _{h \in S_{1}(0)} \sigma_{K}(h) .
$$

3.2. Strong Feller in the Strict Sense Selector under the Structural Stability Assumption. In what follows, we assume that there are no trading constraints, i.e., $D_{t}(\cdot) \equiv \mathbb{R}^{n}$. It turns out that under the assumption of structural stability, i.e., the RNDAO condition, and some stronger assumptions about the multifunctions $K_{t}(\cdot)$, the assertion of [16], Theorem 2, concerning the existence of a Feller kernel selector, can be strengthened; moreover, it can be shown in a constructive manner.

Theorem 1. If the set of initial prices $K_{0}$ is convex and compact, the values of the continuous multifunctions $K_{t}(\cdot)$ are convex compact sets, and RNDAO holds, then there exists a stochastic model, satisfying consistency condition (3), such that the kernels $Q_{t}$ are strong Feller in the strict sense (We follow the terminology of [18] Chapter 1, Definition 5.8. The kernels $Q_{t}$ are iff the mapping $x \longrightarrow Q_{t}(x, \cdot)$ is continuous in the metric on the space of probability measures (equipped with a $\sigma$-algebra), generated by the total variation norm on the space of finite alternating measures).

Proof. Let $\lambda$ be the Lebesgue measure on $\left(\mathbb{R}^{n}, \mathscr{B}^{n}\right)$ and let $Q_{t}, t=1, \ldots, N$ be transition kernels, satisfying consistency condition (3), such that for every $x$, the measure $Q_{t}(x, \cdot)$ is absolutely continuous with respect to $\lambda(\cdot)$ and for every $x$, the (conditional) density equals

$$
q_{t}(x, y)=\frac{I_{K_{t}(x)}(y)}{\lambda\left(K_{t}(x)\right)}, \quad \lambda-\text { a.e. }
$$

The function $r: \mathscr{C}_{\mathbb{R}^{n}}^{0} \longrightarrow \mathbb{R}$ defined by (6) is continuous (even Lipschitz with constant equal to 1; see [19], Lemma 4). Denote

$$
r_{t}^{*}=\min _{x \in B_{t-1}} r\left(K_{t}(x)\right), \quad t=1, \ldots, N
$$

The minimum in (6) is attained because the set of possible trajectories $B_{t-1}$ is compact, since $K_{0}$ is compact and the compact-valued mapping $K_{t}(\cdot)$ is continuous. (For compact-valued mappings, $h$-continuity coincides with continuity, see, e.g., Corollary 2.69 in [20].) The quantity $r_{t}^{*}$ is positive thanks to the RNDSA condition; see [19], Lemma 5. As a consequence, the definition of the densities $q_{t}$ is correct, since 


$$
0<\pi^{n / 2} \Gamma\left(1+\frac{n}{2}\right)\left(r_{t}^{*}\right)^{n} \leq \lambda\left(K_{t}(x)\right) \leq \pi^{n / 2} \Gamma\left(1+\frac{n}{2}\right)\left(\max _{x \in B_{t-1}}\left\|K_{t}(x)\right\|_{2}\right)^{n}<\infty
$$

where $\Gamma$ is the gamma function. Since the compact sets $K_{t}(x)$ are convex, we have (see, e.g., Theorem 14.1 in [21])

$$
d_{\mathrm{PH}}\left(K_{t}\left(x^{\prime}\right), K_{t}(x)\right)=\max _{u \in S_{1}(0)}\left|\sigma_{K_{t}\left(x^{\prime}\right)}(u)-\sigma_{K_{t}(x)}(u)\right| .
$$

Denote this quantity by $\delta\left(x^{\prime}, x\right)$. Therefore, for any $u \in S_{1}(0)$, we have

$$
\sigma_{K_{t}\left(x^{\prime}\right)}(u) \leq \sigma_{K_{t}(x)}(u)+\delta\left(x^{\prime}, x\right) \leq \gamma \sigma_{K_{t}(x)}(u),
$$

$$
\gamma=\gamma\left(x^{\prime}, x\right)=1+\frac{\delta\left(x^{\prime}, x\right)}{r_{t}^{*}}
$$

Similarly, we have

$$
\sigma_{K_{t}(x)}(u) \leq \gamma \sigma_{K_{t}\left(x^{\prime}\right)}(u) .
$$

Thus, (In the general case (for nonconvex sets), if $\sigma_{A}(u) \leq \sigma_{B}(u)$ for all $u \in S_{1}(0)$, then $\mathrm{cl}$ (conv $(A)) \subseteq \operatorname{cl}(\operatorname{conv}(A)).) K_{t}\left(x^{\prime}\right) \subseteq \gamma K_{t}(x)$ and $K_{t}(x) \subseteq \gamma K_{t}\left(x^{\prime}\right)$. Now,

where

$$
\begin{aligned}
\left\|Q_{t}\left(x^{\prime}, \cdot\right)-Q_{t}(x, \cdot)\right\|_{\mathrm{TV}} & =\int\left|q_{t}\left(x^{\prime}, y\right)-q_{t}(x, y)\right| \lambda(\mathrm{d} y) \\
& =\frac{\lambda\left(K_{t}\left(x^{\prime}\right) \backslash K_{t}(x)\right)}{\lambda\left(K_{t}\left(x^{\prime}\right)\right)}+\frac{\lambda\left(K_{t}(x) \backslash K_{t}\left(x^{\prime}\right)\right)}{\lambda\left(K_{t}(x)\right)}+\left|\frac{1}{\lambda\left(K_{t}\left(x^{\prime}\right)\right)}-\frac{1}{\lambda\left(K_{t}(x)\right)}\right| \lambda\left(K_{t}\left(x^{\prime}\right) \cap K_{t}(x)\right) .
\end{aligned}
$$

We have

$$
\lambda\left(K_{t}\left(x^{\prime}\right) \backslash K_{t}(x)\right) \leq \lambda\left(K_{t}\left(x^{\prime}\right) \backslash \gamma^{-1} K_{t}\left(x^{\prime}\right)\right)=\left(1-\gamma^{-1}\right) \lambda\left(K_{t}\left(x^{\prime}\right)\right) .
$$

Similarly, we have

Also,

$$
\lambda\left(K_{t}(x) \backslash K_{t}\left(x^{\prime}\right)\right) \leq\left(1-\gamma^{-1}\right) \lambda\left(K_{t}(x)\right) .
$$

$$
\begin{aligned}
\lambda\left(K_{t}\left(x^{\prime}\right) \cap K_{t}(x)\right) & \leq \lambda\left(K_{t}\left(x^{\prime}\right)\right) \wedge \lambda\left(K_{t}(x)\right) \\
\left|\lambda\left(K_{t}\left(x^{\prime}\right)\right)-\lambda\left(K_{t}(x)\right)\right| & =\left[\lambda\left(K_{t}\left(x^{\prime}\right)\right)-\lambda\left(K_{t}(x)\right)\right] \vee\left[\lambda\left(K_{t}(x)\right)-\lambda\left(K_{t}\left(x^{\prime}\right)\right)\right] \\
& \leq\left(1-\gamma^{-1}\right)\left[\lambda\left(K_{t}\left(x^{\prime}\right)\right) \vee \lambda\left(K_{t}(x)\right)\right] .
\end{aligned}
$$

These inequalities imply

$$
\left\|Q_{t}\left(x^{\prime}, \cdot\right)-Q_{t}(x, \cdot)\right\|_{\mathrm{TV}} \leq\left[3\left(1-\gamma^{-1}\right)\right] \wedge 2=\frac{3 d_{\mathrm{PH}}\left(K_{t}\left(x^{\prime}\right), K_{t}(x)\right)}{d_{\mathrm{PH}}\left(K_{t}\left(x^{\prime}\right), K_{t}(x)\right)+r_{t}^{*}} \wedge 2,
$$

where $r_{t}^{*}$ is defined by (8), and so the kernels $Q_{t}$ are strong Feller in the strict sense.

3.3. Preservation of the Structural Stability for Stochastic Models. Denote by $H_{u}=\left\{y \in \mathbb{R}^{n}:\langle u, y\rangle>0\right\}$ the open half- space with normal vector $u$ and by $B^{\varepsilon}=\left\{z \in \mathbb{R}^{n}: \rho(z, B)<\varepsilon\right\}$ the $\varepsilon$-neighborhood of the set $B$ and consider three metrics on the space of probability measures (Note that $l_{\infty}$ is the minimal metric with respect to the metric on the space of random vectors defined by $L_{\infty}\left(X^{\prime}, X\right)=\operatorname{vrai} \max \rho\left(X^{\prime}, X\right)$, see, e.g., (7.5.15) in [22]. The metric $d_{U C}$ is a generalisation of 
the Kolmogorov metric $d_{K}$ defined for probabilities on the real line $\mathbb{R}$. On $\mathbb{R}$, these two metrics are equivalent: $d_{K} \leq d_{U C} \leq 2 d_{K}$. Note that the metric $d_{U C}$ is used, for example, to estimate the speed of convergence in the multidimensional central limit theorem, see [23]. The Prokhorov metric $d_{P}$ metrises the weak topology on the space of probabilities on a separable metric space.) on $\left(\mathbb{R}^{n}, \mathscr{B}^{n}\right)$ :

$$
\begin{aligned}
d_{U C}\left(Q^{\prime}, Q\right) & =\sup _{A \in \mathscr{C}_{\mathbb{R}^{n}}}\left|Q^{\prime}(A)-Q(A)\right|, \\
l_{\infty}\left(Q^{\prime}, Q\right) & =\inf \left\{\varepsilon>0: Q(B) \leq Q^{\prime}\left(B^{\varepsilon}\right), Q^{\prime}(B) \leq Q\left(B^{\varepsilon}\right), \text { for all } B \in \mathscr{B}^{n}\right\}, \quad \text { and the Prokhorov metric, } \\
d_{P}\left(Q^{\prime}, Q\right) & =\inf \left\{\varepsilon>0: Q(B) \leq Q^{\prime}\left(B^{\varepsilon}\right)+\varepsilon, Q^{\prime}(B) \leq Q\left(B^{\varepsilon}\right)+\varepsilon, \text { for all } B \in \mathscr{B}^{n}\right\} .
\end{aligned}
$$

Theorem 2. Let $Q_{t}$ be the kernels of the initial model and $\widetilde{Q}_{t}$ those of the perturbed model. Assume that for the initial model, RNDAO holds (using consistency condition (3)):

(1) Then, (In this case, the supports $Q_{t}(x, \cdot)$ and $\widetilde{Q}_{t}(x, \cdot)$ are not necessarily compact.) $p_{t}^{*}(x)=\inf _{u \in S_{1}(0)} Q_{t}\left(x, H_{u}\right)>0$, and the perturbed model with kernels $\widetilde{Q}_{t}$ satisfies RNDAO if it is close to the initial one in the sense that

$$
d_{U C}\left(\widetilde{Q}_{t}(x, \cdot), Q_{t}(x, \cdot)\right)<p_{t}^{*}(x) .
$$

(2) Suppose that the supports of $\widetilde{Q}_{t}(x, \cdot)$ and $Q_{t}(x, \cdot)$ are compact. Then, the perturbed model with kernels $\widetilde{Q}_{t}$ satisfies RNDAO if it is close to the initial one in the sense that (The RNDAO condition (for the initial model $)$ is tantamount to $r\left(K_{t}(x)>0\right.$ for all $x$ and $t$.)

$$
l_{\infty}\left(\widetilde{Q}_{t}(x, \cdot), Q_{t}(x, \cdot)\right)<r_{t}^{*}(x),
$$

where $r_{t}^{*}(x)=r\left(K_{t}(x)\right)>0$ and $r$ is given by (6).

(3) There exists a $d_{t}^{*}(x) \in(0,1)$ that can be defined, given the measure $Q_{t}(x, \cdot)$, such that the perturbed model with kernels $\widetilde{Q}_{t}$ satisfies RNDAO if (In this case also, the supports $Q_{t}(x, \cdot)$ and $\widetilde{Q}_{t}(x, \cdot)$ are not necessarily compact.)

$$
d_{P}\left(\widetilde{Q}_{t}(x, \cdot), Q_{t}(x, \cdot)\right)<d_{t}^{*}(x) .
$$

If, additionally, the supports of $Q_{t}(x, \cdot)$ are compact, then it is sufficient to set

$$
d_{t}^{*}(x)=\phi_{t, x}^{\lfloor-1\rfloor}\left(r\left(K_{t}(x)\right)\right),
$$

where $r$ is given by (6),

$$
\begin{aligned}
\phi_{t, x}(z) & =z+\psi_{t, x}^{\lfloor-1\rfloor}(z), \\
\psi_{t, x}(u) & =\inf _{y \in K_{t}(x)} Q\left(x, \bar{B}_{u}(y)\right) .
\end{aligned}
$$

Proof

(1) It is straightforward to see that RNDAO is tantamount to the condition $Q_{t}\left(x, H_{u}\right)>0$ for all $u \in S_{1}(0)$. Consider $O(n)$, the orthogonal group (This is the group of distance-preserving transformations of a Euclidean space of dimension $n$ preserving a fixed point, which we choose equal to 0 . Equivalently, it can be represented as the group of $n \times n$ orthogonal matrices with the group operation given by matrix multiplication.) in dimension $n$. The action of this group of (continuous) transformations is transitive on the unit sphere $S_{1}(0)$, and so if we fix some vector $e \in S_{1}(0)$, then (Given two measurable spaces $(X, \mathscr{A})$ and $(Y, \mathscr{B})$ and an $(\mathscr{A}, \mathscr{B})$-measurable mapping $f: X \longrightarrow Y$, the image of the measure $\pi$ on $(X, \mathscr{A})$ under the mapping $f$ is denoted by $f \circ \pi$ (or $\pi \circ f^{-1}$ ) and is defined by $\left.f \circ \pi(B)=\pi\left(f^{-1}(B)\right), B \in \mathscr{B}.\right)$

$$
\inf _{u \in S_{1}(0)} Q_{t}\left(x, H_{u}\right)=\inf _{\Theta \in O(n)} \Theta \circ Q_{t}\left(x, H_{e}\right) .
$$

Since $O(n)$ is a compact topological group, the mapping $\Theta \mapsto \Theta \circ Q_{t}(x, \cdot)$ is continuous with respect to the weak topology on the space of probability measures. The set $H_{e}$ is open, and hence, by Alexandrov's theorem [24], the function $\Theta \mapsto \Theta \circ Q_{t}\left(x, H_{e}\right)$ is lower semicontinuous and therefore attains a minimum for some $\Theta^{*}=\Theta_{t, x}^{*} \in O(n)$. Denote $u^{*}=\Theta^{*}(e)$; then, $p_{t}^{*}(x)=Q_{t}\left(x, H_{u^{*}}\right)>0$. We have

$$
\begin{aligned}
& \inf _{u \in S_{1}(0)} \widetilde{Q}_{t}\left(x, H_{u}\right)-\inf _{u \in S_{1}(0)} Q_{t}\left(x, H_{u}\right) \mid \\
& \leq \sup _{u \in S_{1}(0)}\left|\widetilde{Q}_{t}\left(x, H_{u}\right)-Q_{t}\left(x, H_{u}\right)\right| \leq \sup _{A \in \mathscr{C}_{\mathbb{R}^{n}}} \mid \widetilde{Q}_{t}(x, A) \\
& \quad-Q_{t}(x, A) \mid=d_{U C}\left(\widetilde{Q}_{t}(x, \cdot), Q_{t}(x, \cdot)\right),
\end{aligned}
$$

whence

$$
\inf _{u \in S_{1}(0)} \widetilde{Q}_{t}\left(x, H_{u}\right) \geq p_{t}^{*}(x)-d_{U C}\left(\widetilde{Q}_{t}(x, \cdot), Q_{t}(x, \cdot)\right)>0,
$$

and thus we have obtained the desired result.

(2) It follows directly from the definition of the metric $l_{\infty}$ that

$$
d_{\mathrm{PH}}\left(\widetilde{K}_{t}(x), K_{t}(x)\right) \leq l_{\infty}\left(\widetilde{Q}_{t}(x, \cdot), Q_{t}(x, \cdot)\right),
$$

where $\widetilde{K}_{t}(x)=\operatorname{supp}\left(\widetilde{Q}_{t}(x, \cdot)\right)$ and $K_{t}(x)$ is defined by (3). The RNDAO condition (for the initial model) implies $r\left(K_{t}(\cdot)\right)>0$. Recall that the function $r: \mathscr{C}_{\mathbb{R}^{n}}^{0} \longrightarrow \mathbb{R}$ is Lipschitz with constant equal to 1 ; 
see Lemma 4 in [19]. Consequently, $\left|r\left(\widetilde{K}_{t}(x)\right)-r\left(K_{t}(x)\right)\right| \leq d_{\mathrm{PH}}\left(\widetilde{K}_{t}(x), K_{t}(x)\right)$; hence,

and we are done.

$$
r\left(\widetilde{K}_{t}(x)\right) \geq r\left(K_{t}(x)\right)-d_{\mathrm{PH}}\left(\widetilde{K}_{t}(x), K_{t}(x)\right) \geq r\left(K_{t}(x)\right)-l_{\infty}\left(\widetilde{Q}_{t}(x, \cdot), Q_{t}(x, \cdot)\right)
$$

Fix some $t$ and $x$. Thanks to the RNDAO condition, $\operatorname{conv}\left(K_{t}(x)\right) \supseteq \bar{B}_{r\left(K_{t}(x)\right)}(0)$. In the case when $K_{t}(x)$ is not finite, choose an arbitrary $\delta \in\left(0, r\left(K_{t}(x)\right)\right.$. There exists a polytope $P_{\delta} \subseteq \bar{B}_{r\left(K_{t}(x)\right)}$ $(0) \subseteq \operatorname{conv}\left(K_{t}(x)\right) \quad$ such that $d_{\mathrm{PH}}\left(P_{\delta}, \bar{B}_{r}\right.$ $\left.\left(K_{t}(x)\right)(0)\right)<\delta$, see, e.g., Theorem 14.6 in [21]. Therefore, $P_{\delta} \supseteq \bar{B}_{r\left(K_{t}(x)\right)-\delta}(0)$. The polytope is the convex hull of a finite set of its vertices, and since each vertex belongs to conv $\left(K_{t}(x)\right)$, it can be represented as a convex combination of a finite set of vectors from $K_{t}(x)$ (in fact, convex combinations of $n+1$ vectors are sufficient by Carathéodory's theorem). Hence, in any case, whether $K_{t}(x)$ is finite or not, there exists (depending on $\delta$ ) a finite set $\left\{y_{1}, \ldots, y_{m}\right\} \subseteq K_{t}(x)$ such that $\operatorname{conv}\left(\left\{y_{1}\right.\right.$, $\left.\left.\ldots, y_{m}\right\}\right) \supseteq \bar{B}_{r\left(K_{t}(x)\right)-\delta}(0)$; clearly, $m \geq n+1$.

Let us introduce two nondecreasing functions (Note that the functions $\zeta_{t, x}$, and therefore $\eta_{t, x}$, depend on the choice of $\delta$.):

$$
\begin{aligned}
& \zeta_{t, x}(u)=\min _{i=1, \ldots, m} Q_{t}\left(x, \bar{B}_{u}\left(y_{i}\right)\right)>0, \\
& \eta_{t, x}(z)=z+\zeta_{t, x}^{\lfloor-1\rfloor}(z) .
\end{aligned}
$$

Suppose that

$$
d_{P}\left(\widetilde{Q}_{t}(x, \cdot), Q_{t}(x, \cdot)\right)<\eta_{t, x}^{\lfloor-1\rfloor}\left(r\left(K_{t}(x)\right)-\delta\right) .
$$

Choose $\delta^{*}>0$ such that

$$
\delta^{*}<\eta_{t, x}^{\lfloor-1\rfloor}\left(r\left(K_{t}(x)\right)-\delta\right)-d_{P}\left(\widetilde{Q}_{t}(x, \cdot), Q_{t}(x, \cdot)\right),
$$

and choose $\varepsilon$ such that

$$
d_{P}\left(\widetilde{Q}_{t}(x, \cdot), Q_{t}(x, \cdot)\right)<\varepsilon<\eta_{t, x}^{\lfloor-1\rfloor}\left(r\left(K_{t}(x)\right)-\delta\right)-\delta^{*}
$$

Since $\quad \varepsilon>d_{P}\left(\widetilde{Q}_{t}(x, \cdot), Q_{t}(x, \cdot)\right)$, we have $\widetilde{Q}_{t}\left(x, \bar{B}_{u+\varepsilon}(y)\right) \geq Q_{t}\left(x, \bar{B}_{u}(y)\right)-\varepsilon$ for any $y \in K_{t}(x)$ and arbitrary $u \geq 0$. Therefore, $\widetilde{Q}_{t}\left(x, \bar{B}_{u+\varepsilon}\left(y_{i}\right) \geq \zeta_{t, x}(u)\right)-\varepsilon$ for every $i=1, \ldots, m$. If we choose $u$ such that $u \in\left(\zeta_{t, x}^{\lfloor-1\rfloor}(z), \zeta_{t, x}^{\lfloor-1\rfloor}(z)+\delta^{\prime}\right)$, where $\delta^{\prime}<\delta^{*}$, then $\zeta_{t, x}(u)>\varepsilon$, and $\widetilde{K}_{t}(x) \cap \bar{B}_{u+\varepsilon}\left(y_{i}\right) \neq \varnothing$ for any $i=1, \ldots, m$, and so we can choose $\widetilde{y}_{i} \in \widetilde{K}_{t}(x) \cap \bar{B}_{u+\varepsilon}\left(y_{i}\right), i=1, \ldots, m$. By the choice of $\varepsilon$ and $u$, we obtain

$$
r\left(K_{t}(x)\right)-\delta>\eta\left(\varepsilon+\delta^{*}\right) \geq \varepsilon+\zeta_{t, x}^{\lfloor-1\rfloor}(\varepsilon)+\delta^{*} \geq u+\varepsilon .
$$

Therefore, $r\left(K_{t}(x)\right)-\delta-u-\varepsilon>0$ and

$$
\operatorname{conv}\left(\widetilde{K}_{t}(x)\right) \supseteq \operatorname{conv}\left(\left\{\widetilde{y}_{1}, \ldots, \widetilde{y}_{m}\right\}\right) \supseteq B_{r\left(K_{t}(x)\right)-\delta-u-\varepsilon}(0) .
$$

Thus, RNDAO holds for the perturbed model when $d_{P}\left(\widetilde{Q}_{t}(x, \cdot), Q_{t}(x, \cdot)\right)<d_{t}^{*}(x), \quad$ where $\quad d_{t}^{*}(x)=\eta_{t, x}^{\lfloor-1\rfloor}(r$ $\left.\left(K_{t}(x)\right)-\delta\right)$.

Now, consider the case when $\operatorname{supp}\left(K_{t}(x)\right)$ is compact. The function $y \mapsto Q_{t}\left(x, B_{u}(y)\right)>0$ is lower semicontinuous, thanks to Alexandrov's theorem, and so $\inf _{y \in K_{t}(x)} Q\left(x, B_{u}(y)\right)>0$ for $u>0$. Note that for the function $\psi_{t, x}$ defined in the statement of part 3 of Theorem 2, we have $\psi_{t, x}(u)>0$ for $u>0$. Since $y \mapsto Q_{t}\left(x, \bar{B}_{u}(y)\right)>0$ is upper semicontinuous, $\psi_{t, x}$ is also upper semicontinuous; moreover, it is nondecreasing and hence right-continuous, and $\psi_{t, x}(u)=1$ for sufficiently large values of $u$. For small positive values of $u$, we have

$$
\begin{aligned}
\psi_{t, x}(0) & =\lim _{u \downarrow 0} \psi_{t, x}(u)=\inf _{u>0} \inf _{y \in K_{t}(x)} Q_{t}\left(x, \bar{B}_{u}(y)\right) \\
& =\inf _{y \in K_{t}(x)} \inf _{u>0} Q_{t}\left(x, \bar{B}_{u}(y)\right)=\inf _{y \in K_{t}(x)} Q_{t}(x,\{y\}) .
\end{aligned}
$$

Consequently, $\psi_{t, x}(0)$ is positive iff $K_{t}(x)$ is a finite set and zero otherwise. Note that the function $\phi_{t, x}$ defined in the statement of part 3 of Theorem 2 is strictly increasing, and hence $\phi_{t, x}^{\lfloor-1\rfloor}(\cdot)$ is continuous, $\phi_{t, x}^{\lfloor-1\rfloor}(0)=0$, and $\phi_{t, x}^{\lfloor-1\rfloor}(u)>0$ for $u>0$. The RNDAO condition for the initial model is tantamount to $r\left(K_{t}(\cdot)\right)>0$, and hence, $\phi_{t, x}^{-1}\left(r\left(K_{t}(x)\right)\right)>0$. If $d_{P}\left(\widetilde{Q}_{t}(x, \cdot), Q_{t}(x, \cdot)\right)<\phi_{t, x}^{\lfloor-1\rfloor}\left(r\left(K_{t}(x)\right)\right)$, choose $\delta^{*}>0$ such that $\delta^{\star}<\phi^{\lfloor-1\rfloor}\left(r\left(K_{t}(x)\right)\right)-d_{P}\left(\widetilde{Q}_{t}(x, \cdot), Q_{t}(x, \cdot)\right) \quad$ and choose $\varepsilon_{0}$ such that

$$
d_{P}\left(\widetilde{Q}_{t}(x, \cdot), Q_{t}(x, \cdot)\right)<\varepsilon_{0}<\phi^{\lfloor-1\rfloor}\left(r\left(K_{t}(x)\right)\right)-\delta^{*} .
$$

Denote $\delta_{0}=u-\psi_{t, x}^{\lfloor-1\rfloor}\left(\varepsilon_{0}\right)$; if $\delta_{0}>0$, i.e., $u>\psi_{t, x}^{\lfloor-1\rfloor}\left(\varepsilon_{0}\right)$, then $\psi_{t, x}(u)>\varepsilon_{0}$ and $\bar{Q}_{t}\left(x, \bar{B}_{u+\varepsilon_{0}}(y)\right) \geq \psi_{t, x}(u)-\varepsilon_{0}>0$ for any $y \in K_{t}(x)$, whence $\widetilde{K}_{t}(x) \cap \bar{B}_{u+\varepsilon_{0}}(y) \neq \varnothing$ for any $y \in K_{t}(x)$. Recall that for arbitrary $\delta>0$, there exists (depending on $\delta$ ) a finite set $\left\{y_{1}, \ldots, y_{m}\right\} \subseteq K_{t}(x)$ such that $\operatorname{conv}\left(\left\{y_{1}, \ldots, y_{m}\right\}\right) \supseteq \bar{B}_{r\left(K_{t}(x)\right)-\delta}(0)$; choose $\delta>0$ and $\delta_{0}>0$ such that $\delta+\delta_{0}<\delta^{\star}$. Then, we can choose (depending on $\delta$ ) points $\widetilde{y}_{i} \in \widetilde{K}_{t}(x) \cap \bar{B}_{u+\varepsilon_{0}}\left(y_{i}\right), i=1, \ldots, m$. Thanks to our choice of parameters, we have

$$
r\left(K_{t}(x)\right)>\phi\left(\varepsilon_{0}+\delta^{*}\right) \geq \delta^{*}+\varepsilon_{0}+\psi_{t, x}^{\lfloor-1\rfloor}\left(\varepsilon_{0}\right)>\delta+u+\varepsilon_{0} .
$$

Therefore, $r\left(K_{t}(x)\right)-\delta-u-\varepsilon_{0}>0$ and $\operatorname{conv}\left(\widetilde{K}_{t}(x)\right) \supseteq \operatorname{conv}\left(\left\{\widetilde{y}_{1}, \ldots, \tilde{y}_{m}\right\}\right) \supseteq B_{r\left(K_{t}(x)\right)-\delta-u-\varepsilon_{0}}(0)$. Thus, RNDAO holds for the perturbed model, when $d_{P}\left(\widetilde{Q}_{t}(x, \cdot), Q_{t}(x, \cdot)\right)<d_{t}^{*}(x), \quad$ where $\quad d_{t}^{*}(x)=\phi_{t, x}^{\lfloor-1\rfloor}$ $\left(r\left(K_{t}(x)\right)\right)$. 
Remark 1. Note that the metric $d_{U C}$ generates stronger convergence than the weak one: this follows from Example 2.3 in [25]. On the other hand, $d_{U C}\left(Q^{\prime}, Q\right)$ $\leq(1 / 2)\left\|Q^{\prime}-Q\right\|_{T V}$, and the convergence in the total variation norm is stronger. Therefore, the result of [4] follows from part 1 of Theorem 2; moreover, our proof is shorter. Note also that the Prokhorov metric $d_{P} \leq l_{\infty}$. The convergence in the $l_{\infty}$ metric implies weak convergence (for a separable metric space) and is stronger.

\section{Conclusions}

Depending on the context, structural stability can be formalised in different ways. We propose a formalisation of the structural stability of the financial market model in the deterministic framework. It turns out that this approach also works for stochastic models. Under the assumption of structural stability, we construct a transition kernel selector, satisfying the strict Feller property. We show the preservation of structural stability for transitional kernel perturbations, small enough with respect to one of the three probability metrics considered. This is one more argument in favour of the importance of structural stability for financial modelling.

\section{Data Availability}

No data were used to support this study.

\section{Conflicts of Interest}

The author declares that there are no conflicts of interest regarding the publication of this paper.

\section{Acknowledgments}

This work was supported by the Russian Foundation for Basic Research (research projects 19-01-00613 a and 16-2904191 ofi $\mathrm{m})$.

\section{References}

[1] A. A. Andronov and L.S. Pontryagin, "Systèmes grossiers," Doklady Akademii Nauk SSSR, vol. 14, pp. 247-250, 1937.

[2] W. Schachermayer, "The fundamental theorem of asset pricing under proportional transaction costs in finite discrete time," Mathematical Finance, vol. 14, no. 1, pp. 19-48, 2004.

[3] E. Bayraktar, Y. Zhang, and Z. Zhou, "A note on the fundamental theorem of asset pricing under model uncertainty," Risks, vol. 2, no. 4, pp. 425-433, 2014.

[4] V. Ostrovski, "Stability of no-arbitrage property under model uncertainty," Statistics \& Probability Letters, vol. 83, no. 1, pp. 89-92, 2013.

[5] Z. Hou and J. Obłój, "Robust pricing-hedging dualities in continuous time," Finance and Stochastics, vol. 22, no. 3, pp. 511-567, 2018.

[6] S. N. Smirnov, "Guaranteed deterministic approach to superhedging: sensitivity of solutions of the bellman-isaacs equations and numerical methods," Computational Mathematics and Modeling, vol. 31, no. 3, pp. 384-401, 2020.
[7] L. Carassus and T. Vargiolu, "Super-replication price: it can be OK," ESAIM: Proceedings and Surveys, vol. 65, pp. 241-281, 2018.

[8] V.N. Kolokoltsov, "Nonexpansive maps and option pricing theory," Kybernetika, vol. 34, pp. 713-724, 1998.

[9] P. Bernhard, J. C. Engwerda, B. Roorda et al., The Interval Market Model in Mathematical Finance: Game-Theoretic Methods, Springer, Berlin, Germany, 2013.

[10] B. Bouchard and M. Nutz, "Arbitrage and duality in nondominated discrete-time models," Annals of Applied Probability, vol. 25, pp. 823-859, 2015.

[11] M. Burzoni, M. Frittelli, Z. Hou et al., "Pointwise arbitrage pricing theory in discrete time," Mathematics of Operations Research, vol. 44, pp. 1034-1057, 2019.

[12] J. Oblój and J. Wiesel, "A unified framework for robust modelling of financial markets in discrete time," 2019, http:// arxiv.org/abs/1808.06430v2.

[13] D. Dubois and H. Prade, Fuzzy Sets and Systems: Theory and Applications, Academic Press, New York, NY, USA, 1980.

[14] S. N. Smirnov, "Geometric criterion for a robust condition of no sure arbitrage with unlimited profit," Moscow University Computational Mathematics and Cybernetics, vol. 44, pp. 146-150, 2020.

[15] J. Jacod and A.N. Shiryaev, "Local martingales and the fundamental asset pricing theorems in the discrete-time case," Finance and Stochastics, vol. 2, pp. 259-273, 1998.

[16] S.N. Smirnov, "A feller transition kernel with measure supports given by a set-valued mapping," Proceedings of the Steklov Institute of Mathematics, vol. 308, pp. 188-195, 2020.

[17] C. T. Ionescu Tulcea, "Mesures dans les espaces produits," Atti della Accademia Nazionale dei Lincei Rendiconti, vol. 7, pp. 208-211, 1949.

[18] D. Revuz, Markov Chains, Springer, Amsterdam, The Netherlands, 1975.

[19] S. N. Smirnov, "The guaranteed deterministic approach to superhedging: Lipschitz properties of solutions of the Bellman-Isaacs equations," in Frontiers of Dynamics Games: Game Theory and Management, pp. 267-288, Birkhäuser, St. Petersburg, Russia, 2019.

[20] S. Hu and N. Papageorgiou, "Handbook of multivalued analysis: Theory," Mathematics and its Applications, Vol. 1, Springer, Berlin, Germany, 1997.

[21] K. Leichtweiß, Konvexe Mengen, Springer-Verlag, Berlin, Germany, 1979.

[22] S. Rachev, L. Klebanov, S. Stoyanov et al., The Methods of Distances in the Theory of Probability and Statistics, SpringerVerlag, Berlin, Germany, 2013.

[23] R. Bentkus, "“On the dependence of the Berry-Esseen bound on dimension," Journal of Statistical Planning and Inference, vol. 113, pp. 385-402, 2003.

[24] A. D. Alexandroff, "Additive set-functions in abstract spaces," Recueil Mathématique [Mat. Sbornik] N.S.vol. 13, no. 55, pp. 169-238, 1943.

[25] P. Billingsley, Convergence of Probability Measures, Wiley, New York, NY, USA, 2nd edition, 1999. 